\title{
The Michigan Appropriateness Guide for Intravenous Catheters (MAGIC) Initiative: A Summary and Review of Peripherally Inserted Central Catheter and Venous Catheter Appropriate Use
}

\author{
Scott C. Woller, MD ${ }^{1,2 *}$, Scott M. Stevens, MD ${ }^{1,2}$, R. Scott Evans, MS, PhD, FACMl ${ }^{3}$
}

${ }^{1}$ Department of Medicine, Intermountain Medical Center, Murray, Utah; '2Department of Internal Medicine, University of Utah, Salt Lake City, Utah; ${ }_{3}^{3}$ Medical Informatics, Intermountain Healthcare, Biomedical Informatics, University of Utah, Salt Lake City, Utah.

Peripherally inserted central catheters (PICCs) are being selected for venous access more frequently today than ever before. Often the choice of a PICC, when compared with other vascular access devices (VADs), is attractive because of perceived safety, availability, and ease of insertion. However, complications associated with PICCs exist, and there is a paucity of evidence to guide clinician choice for PICC selection and valid use. An international panel with expertise in the arena of venous access and populations associated with these devices was convened to clarify approaches for the optimal use of PICCs and VADs. Here we present for the busy hospital-based practitioner the methodology, key outcomes, and recommendations of the Michigan Appropriateness Guide for Intravenous Catheters (MAGIC) panelists for the appropriate use of VADs. Journal of Hospital Medicine 2016;11:306-310. (C) 2015 Society of Hospital Medicine
Vascular access devices (VADs), including peripherally inserted central venous catheters (PICCs) and traditional central venous catheters (CVCs), remain a cornerstone for the delivery of necessary therapy. VADs are used routinely to treat inpatients and increasingly outpatients too. PICCs possess characteristics that are often favorable in a variety of clinical settings when compared to traditional CVCs. However, a paucity of evidence regarding the indication, selection, application, duration, and risks associated with these devices exists. PICCs are often used in situations when peripheral venous catheters (PIVs-including ultrasoundguided peripheral intravenous catheters and midline catheters [midlines]) would meet patient needs and confer a lower risk of complications. An unmet need to define indications and promote utilization that conforms to optimal use currently exists. The purpose of this article was to highlight for hospitalists the methodology and subsequent key recommendations published recently ${ }^{1}$ regarding appropriateness of PICCs as they pertain to other vascular access device use.

\section{BACKGROUND}

Greater utilization of PICCs to meet a variety of clinical needs has recently emerged in hospital-based medicine. $^{2,3}$ This phenomenon is likely a function of

*Address for correspondence and reprint requests: Scott C. Woller, MD, Department of Medicine, Intermountain Medical Center, 5121 Cottonwood Street, Murray, UT 84157-7000; Telephone: 801-507-3747; Fax: 801-507-3799; E-mail: scott.woller@imail.org

Additional Supporting Information may be found in the online version of this article.

Received: August 4, 2015; Revised: November 5, 2015; Accepted: November 15, 2015

2015 Society of Hospital Medicine DOI 10.1002/jhm.2525

Published online in Wiley Online Library (Wileyonlinelibrary.com). favorable characteristics when comparing PICCs with traditional CVCs. PICCs are often favored because of safety with insertion in the arm, compatibility with inpatient and outpatient therapies, ease of protocolization for insertion by vascular access nursing services, patient tolerability, and cost savings. ${ }^{4-8}$ Yet limitations of PICCs exist and complications including malpositioning, dislodgement, and luminal occlusion ${ }^{9-11}$ affect patient safety and outcomes. Most notably, PICCs are strongly associated with risk for thrombosis and infection, complications that are most frequent in hospitalized and critically ill patients. ${ }^{12-16}$

Vascular access devices and particularly PICCs pose a substantial risk for thrombosis. ${ }^{16-20}$ PICCs represent the greatest risk factor for upper extremity deep vein thrombosis (DVT), and in one study, PICC-associated DVT risk was double that with traditional CVCs. ${ }^{17}$ Risk factors for the development of PICC-associated DVT include ipsilateral paresis, ${ }^{21}$ infection, ${ }^{22}$ PICC diameter, ${ }^{19,20}$ and prolonged surgery (procedure duration $>1$ hour) with a PICC in place. ${ }^{23}$ Recently, PICCs placed in the upper extremity have been described as a possible risk factor for lower extremity venous thrombosis as well. ${ }^{24,25}$

Infection complicating CVCs is well described, ${ }^{12,15}$ and guidelines for the prevention of catheter-associated blood stream infections exist. ${ }^{26,27}$ However, the magnitude of the risk of infection associated with PICCs compared with traditional CVCs remains uncertain. Some reports suggest a decrease risk for infection with the utilization of $\mathrm{PICCs}^{28}$; others suggest a similar risk. ${ }^{29}$ Existing guidelines, however, do not recommend substituting PICCs for CVCs as a technique to reduce infection, especially in general medical patients. ${ }^{30}$

It is not surprising that variability in the clinical use of PICCs and inappropriate PICC utilization has been 
TABLE 1. Guide for PICC Use

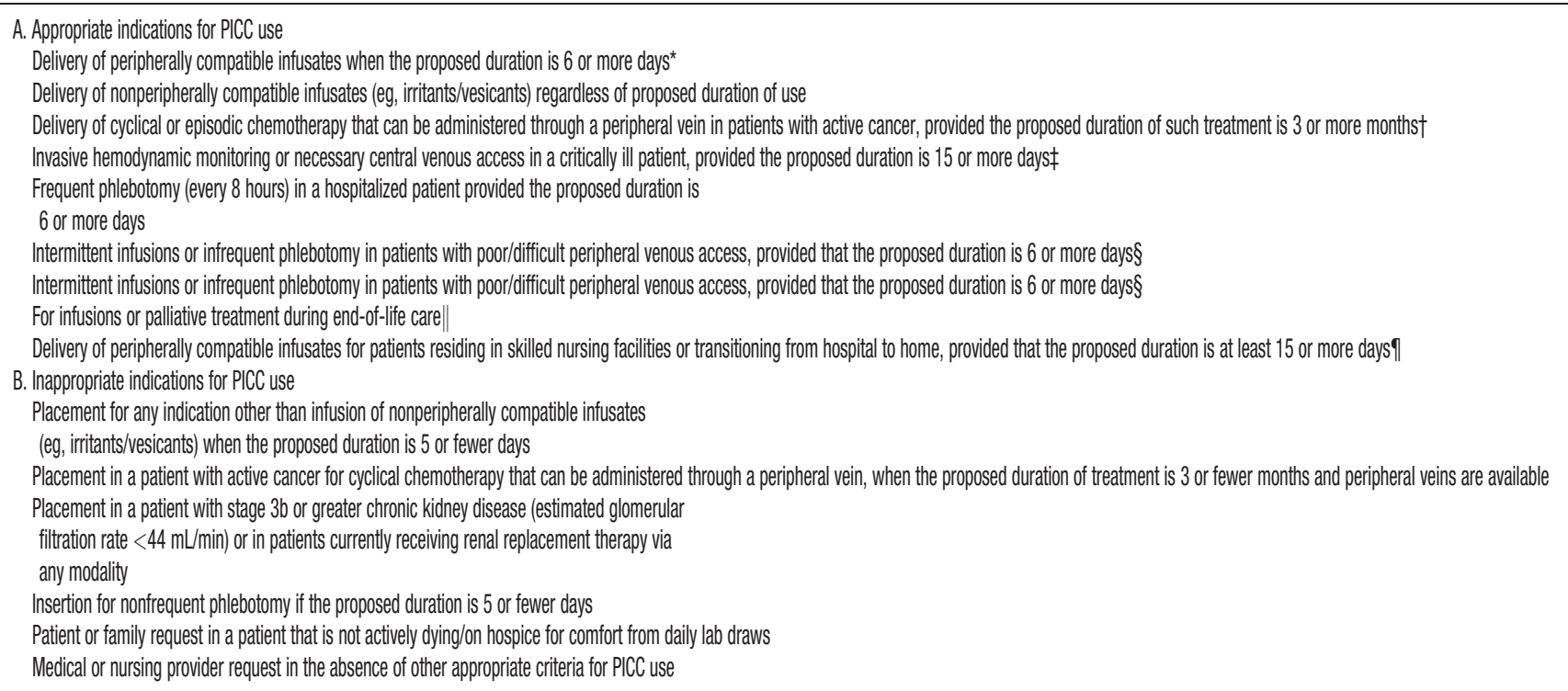

NOTE: Table is derived from Chopra et al. ${ }^{1}$ Abbreviations: PICC, peripherally inserted central catheters. ${ }^{*}$ Use of ultrasound-guided peripheral intravenous catheters or midlines is preferred over use of PICCs for infusion of peripherally compatible infusates up to 14 days. In patients with poor peripheral venous access, use of ultrasound-guided peripheral intravenous catheters and midlines is also preferred over use of PICCs. †In patients with cancer, the risk of thrombosis associated with PICCs may outweigh benefits. Patients who are scheduled to receive multiple cycles of peripherally compatible chemotherapy for durations $<3$ months should do so via peripheral intravenous catheters with each infusion. fUse of nontunneled central venous catheters is preferred over use of PICCs for central venous access or invasive hemodynamic monitoring $<14$ days and in patients with documented hemodynamic instability where urgent venous access is necessary. §Use of ultrasound-guided peripheral intravenous catheters or midlines is preferred over use of PICCs for patients with poor/difficult peripheral venous access. \|Placement of a PICC in a terminally ill patient is appropriate if it facilitates comfort goals of care. PICCs may be left in place in such patients to attain similar goals. ๆUse of PICCs for home-based infusions or in skilled nursing facilities (where resources are limited) is inappropriate for the short-term ( $<14$ days). In such settings, use of peripheral intravenous catheters or midlines is rated as appropriate.

described $^{31,32}$ given the heterogeneity of patients and clinical situations in which PICCs are used. Simple awareness of medical devices in place is central to optimizing care. Important to the hospitalist physician is a recent study that found that 1 in 5 physicians were unaware of a CVC being present in their patient. ${ }^{33}$ Indeed, emphasis has been placed on optimizing the use of PICC lines nationally through the Choosing Wisely initiative. ${ }^{34,35}$

A panel of experts was convened at the University of Michigan in an effort to further clarify the appropriate use of VADs. Panelists engaged in a RAND Corporation/University of California Los Angeles (RAND/ UCLA) Appropriateness Methodology review ${ }^{36}$ to provide guidance regarding VAD use. The RAND/UCLA methodology is a validated way to assess the appropriateness of medical and surgical resource utilization, and details of this methodology are published elsewhere. ${ }^{1}$ In brief, each panelist was provided a series of clinical scenarios associated with the use of central venous catheters purposefully including areas of consensus, controversy, and ambiguity. Using a standardized method for rating appropriateness, whereby median ratings on opposite ends of a 1 to 9 scale were used to indicate preference of one device over another (for example -9 reflected appropriate and 1-3 reflected inappropriate), the methodology classified consensus results into three levels of appropriateness. These three levels are: appropriate when the panel median is between 7 and 9 and without disagreement, uncertain/neutral when the panel median is between 4 and 6 or disagreement exists regardless of the median, or inappropriate when the panel median is between 1 and 3 without disagreement.

\section{RESULTS}

Comprehensive results regarding appropriateness ratings are reported elsewhere. ${ }^{1}$ Results especially key to hospital-based practitioners are summarized below. Table 1 highlights common scenarios when PICC placement is considered appropriate and inappropriate.

\section{Appropriateness of PICCs in General Hospitalized Medical Patients}

The appropriateness of PICCs when compared to other VADs among hospitalized medical patients can be broadly characterized based upon the planned infusate and the anticipated duration of use. PICCs were the preferred VAD when the anticipated duration of infusion was greater than 15 days or for any duration if the infusion was an irritant/vesicant (such as parenteral nutrition or chemotherapy). PICCs were considered appropriate if the proposed duration of use was 6 to 14 days, though preference for a midline or an ultrasoundguided PIV was noted for this time-frame. Tunneled catheters were considered appropriate only for the infusion of an irritant/vesicant when the anticipated duration was $\geq 15$ days; similarly, implanted ports were rated as appropriate when an irritant/vesicant infusion was planned for $\geq 31$ days. Both tunneled catheters and ports were rated as appropriate when episodic infusion over the duration of several months was necessary. 
Disagreement existed between the panelists regarding the appropriateness of PICC placement for the indication of frequent blood draws $(\geq 3$ phlebotomies per day) and among patients with difficult venous access, when phlebotomy would be needed for $\leq 5$ days. In these cases an individualized patient-centered approach was recommended. PICC placement was considered appropriate in these situations if venous access was required $\geq 6$ days, but ultrasound-guided and midline PIVs were again preferred to PICCs when the expected duration of use was $<14$ days.

\section{Appropriateness of PICCs in Patients With Chronic Kidney Disease}

The appropriateness of PICC use among patients with chronic kidney disease (CKD) takes into consideration disease stage as defined by the Kidney Disease: Improving Global Outcomes workgroup. ${ }^{37}$ Although panelist recommendations did not differ for patients with stage 1 to $3 \mathrm{a}$ CKD (estimated GFR $\geq 45 \mathrm{~mL} /$ $\mathrm{min}$ ) from those noted above, for patient's stage $3 \mathrm{~b}$ or greater $\mathrm{CKD}$, insertion of devices into an arm vein was rated as inappropriate (valuing the preservation of peripheral and central veins for possible hemodialysis/creation of arteriovenous fistulae and grafts). Among patients with stage $3 \mathrm{~b}$ or greater CKD, PIV access in the dorsum of the hand was recommended for an expected duration of use $\leq 5$ days. In consultation with a nephrologist, the use of a tunneled smallbore central catheter ( 4 French or 5 French) inserted into the jugular vein was rated as appropriate in stage $3 \mathrm{~b}$ or greater CKD patients requiring venous access for a longer duration.

\section{Appropriateness of PICC Use in Patients with Cancer}

The panelists' acknowledged the heterogeneity of thrombosis risk based on cancer type; recommendations reflect the assumption of cancer as a solid tumor. Vascular access choice among cancer patients is complicated by the cyclic nature of therapy frequently administered, the diversity of infusate (eg, nonirritant or nonvesicant versus irritant/vesicant), and uncertainties surrounding duration of therapy. To address this, the panelists chose a pragmatic approach considering the infusate (irritant/vesicant or not), and dichotomized treatment duration ( $\geq 3$ months or not). Among cancer patients requiring nonvesicant/nonirritant chemotherapy for a duration $\leq 3$ months, interval placement of PIVs was rated as appropriate, and disagreement existed among the panelists regarding the appropriateness of PICCs. If $\geq 3$ months of chemotherapy was necessary, then PICCs or tunneled-cuffed catheters were considered appropriate. Ports were rated as appropriate if the expected use was $\geq 6$ months. Among cancer patients requiring vesicant/irritant chemotherapy, PICCs and tunneled-cuffed catheters were rated as appropriate for all time intervals, and ports were rated as neutral for 3- to 6-month durations of infusion, and appropriate for durations greater than 6 months. When acceptable, PICCs were favored over tunneled-cuffed catheters among cancer patients with coagulopathy (eg, severe thrombocytopenia, elevated international normalized ratios).

\section{Appropriateness of PICCs in Patients With Critical Illness}

Among critically ill patients, PIVs and midline catheters were rated as appropriate for infusion of $\leq 5$ days, and 6 to 14 days, respectively, whereas PICCs were considered appropriate only when use $\geq 15$ days was anticipated. Although both CVCs and PICCs were rated as appropriate among hemodynamically unstable patients in scenarios where invasive cardiovascular monitoring is necessary for durations of $\leq 14$ days and $\geq 15$ days, respectively, CVCs were favored over PICCs among patients who are hemodynamically unstable or requiring vasopressors.

\section{Appropriateness of PICC Use In "Special" Populations}

The existence of patients who require lifelong, often intermittent, intravenous access (eg, sickle cell anemia, short-gut syndrome, cystic fibrosis) necessitates distinct recommendations for venous access. In this population, recommendations were categorized based on frequency of hospitalization. In patients that were hospitalized infrequently $(<5$ hospitalizations per year), use of midlines was preferred to PICCs when the hospitalization was expected to last $\leq 5$ days; PICCs were rated as appropriate for a duration of use $\geq 15$ days. However, in patients who require frequent hospitalization ( $\geq 6$ hospitalizations annually), tunneled-cuffed catheters were rated as appropriate and preferred over PICCs when the expected duration of use was $\geq 15$ days per session.

For long-term residents in skilled nursing facilities, PICCs were rated as appropriate for an expected duration of use $\geq 15$ days, but uncertain for a duration of 6 to 14 days (when midlines were rated as appropriate). For venous access of $\leq 5$ days, PIVs were rated as most appropriate.

\section{How, When, by Whom, and Which PICCs Should Be Inserted}

Societal recommendations ${ }^{26}$ and guidelines ${ }^{38}$ for routine placement and positioning of PICCs by dedicated nursing services exist. ${ }^{39,40}$ Panelists favored consultation with the specialists ordering vascular access devices (eg, infectious disease, nephrology, hematology, oncology) within the first few days of admission for optimal device selection and timing of insertion. For example, PICCs were rated as appropriate to be placed within 2 to 3 days of hospital admission for patients requiring longterm antimicrobial infusion (in the absence of bacteremia). Preferential PICC placement by 
interventional radiology was rated as appropriate if portable ultrasound did not identify a suitable target vein, the catheter fails to advance over the guidewire during a bedside attempt, or the patient requires sedation not appropriate for bedside placement. Interventional radiology insertion was also preferred in patients with bilateral mastectomy, altered chest anatomy, and for patients with permanent pacemakers or defibrillators if the contralateral arm is was not amenable for insertion. PICCs are generally placed at the bedside (with radiographic confirmation of catheter position, or with electrocardiography guidance when proficiency with this technique exists) or under direct visualization in the interventional radiology suite. As recommended elsewhere, ${ }^{21,26,41}$ panelists rated the placement of the PICC catheter tip in the lower one-third of the superior vena cava, at the cavoatrial junction, or in the right atrium as being appropriate. Nuanced recommendations surrounding PICC adjustment under varying circumstances can be found in the parent document. ${ }^{1}$ Single-lumen devices, which are associated with fewer complications, were rated as the appropriate default lumen of choice in the absence of a documented rationale for a multilumen PICC as a mechanism to decrease possible complications. ${ }^{19,20,42}$ The insertion of multilumen PICCs for separating blood draws from infusions or ensuring a "backup" lumen is available was rated as inappropriate. Consistent with recent recommendations, ${ }^{43,44}$ normal saline rather than heparin was rated as appropriate to maintain catheter patency. The advancement of a migrated PICC was rated as inappropriate under all circumstances.

\section{CONCLUSIONS}

In-hospital healthcare providers are routinely confronted with dilemmas surrounding choice of VAD. The Michigan Appropriateness Guide for Intravenous Catheters (MAGIC) initiative is a multidisciplinary effort to clarify decision-making related to VAD use. The systematic literature review and RAND/UCLA appropriateness method applied by the MAGIC panelists identifies areas of broad consensus surrounding the use of PICCs in relation to other VADs, and highlights uncertainties regarding the best practice to guide clinical care. Appropriateness statements facilitate standardization for the use, care, and discontinuation of VADs. These recommendations may be important to healthcare quality officers and payers as they allow for measurement of, and adherence to, standardized practice. In an era of electronic medical records and embedded clinical decision support, these recommendations may facilitate a just-in-time resource for optimal VAD management, outcomes measurement, and patient follow-up. In addition to directing clinical care, these recommendations may serve as a lattice for the formation of future randomized clinical trials to further clarify important areas of the uncertainty surrounding VAD use.
Disclosures: Drs. Woller and Stevens disclose financial support paid to their institution of employment (Intermountain Medical Center) for conducting clinical research (with no financial support paid to either investigator). Dr. Woller discloses serving as an expert panelist for the Michigan Appropriateness Guide for Intravenous Catheters (MAGIC) initiative. The authors report no other conflicts of interest.

\section{References}

1. Chopra V, Flanders SA, Saint S, et al. The Michigan Appropriateness Guide for Intravenous Catheters (MAGIC): results from a multispecialty panel using the RAND/UCLA appropriateness method. Ann Intern Med. 2015;163(6 suppl):S1-S40.

2. Al Raiy B, Fakih MG, Bryan-Nomides N, et al. Peripherally inserted central venous catheters in the acute care setting: a safe alternative to high-risk short-term central venous catheters. Am J Infect Control. 2010;38(2):149-153.

3. Gunst M, Matsushima K, Vanek S, Gunst R, Shafi S, Frankel H. Peripherally inserted central catheters may lower the incidence of catheter-related blood stream infections in patients in surgical intensive care units. Surg Infect (Larchmt). 2011;12(4):279-282.

4. Meyer BM. Developing an alternative workflow model for peripherally inserted central catheter placement. J Infus Nurs. 2012;35(1):3442.

5. Walker G, Todd A. Nurse-led PICC insertion: is it cost effective? $\mathrm{Br} J$ Nurs. 2013;22(19):S9-S15.

6. Burns T, Lamberth B. Facility wide benefits of radiology vascular access teams, part 2. Radiol Manage. 2010;32(3):39-43.

7. Burns T, Lamberth B. Facility wide benefits of radiology vascular access teams. Radiol Manage. 2010;32(1):28-32; quiz 3-4.

8. Johansson E, Hammarskjold F, Lundberg D, Arnlind MH. Advantages and disadvantages of peripherally inserted central venous catheters (PICC) compared to other central venous lines: a systematic review of the literature. Acta Oncol. 2013;52(5):886-892.

9. Chopra V, Flanders SA, Saint S. The problem with peripherally inserted central catheters. JAMA. 2012;308(15):1527-1528.

10. Song L, Li H. Malposition of peripherally inserted central catheter: experience from 3,012 patients with cancer. Exp Ther Med. 2013; 6(4):891-893

11. Pikwer A, Akeson J, Lindgren S. Complications associated with peripheral or central routes for central venous cannulation. Anaesthesia. 2012;67(1):65-71.

12. Chopra V, Anand S, Krein SL, Chenoweth C, Saint S. Bloodstream infection, venous thrombosis, and peripherally inserted central catheters: reappraising the evidence. Am J Med. 2012;125(8):733-741.

13. Li J, Fan YY, Xin MZ, et al. A randomised, controlled trial comparing the long-term effects of peripherally inserted central catheter placement in chemotherapy patients using B-mode ultrasound with modified Seldinger technique versus blind puncture. Eur J Oncol Nurs. 2014;18(1):94-103.

14. Leung TK, Lee CM, Tai CJ, Liang YL, Lin CC. A retrospective study on the long-term placement of peripherally inserted central catheters and the importance of nursing care and education. Cancer Nurs. 2011;34(1):E25-E30.

15. Chopra V, O'Horo JC, Rogers MA, Maki DG, Safdar N. The risk of bloodstream infection associated with peripherally inserted central catheters compared with central venous catheters in adults: a systematic review and meta-analysis. Infect Control Hosp Epidemiol. 2013; 34(9):908-918.

16. Chopra V, Anand S, Hickner A, et al. Risk of venous thromboembolism associated with peripherally inserted central catheters: a systematic review and meta-analysis. Lancet. 2013;382(9889):311-325.

17. Saber W, Moua T, Williams EC, et al. Risk factors for catheterrelated thrombosis (CRT) in cancer patients: a patient-level data (IPD) meta-analysis of clinical trials and prospective studies. J Thromb Haemost. 2011;9(2):312-319.

18. Spencer FA, Emery C, Lessard D, Goldberg RJ. Upper extremity deep vein thrombosis: a community-based perspective. Am J Med. 2007; 120(8):678-684.

19. Evans RS, Sharp JH, Linford LH, et al. Risk of symptomatic DVT associated with peripherally inserted central catheters. Chest. 2010; 138(4):803-810.

20. Evans RS, Sharp JH, Linford LH, et al. Reduction of peripherally inserted central catheter associated deep venous thrombosis. Chest. 2013;143(3):627-633.

21. Wilson TJ, Brown DL, Meurer WJ, Stetler WR Jr, Wilkinson DA, Fletcher JJ. Risk factors associated with peripherally inserted central venous catheter-related large vein thrombosis in neurological intensive care patients. Intensive Care Med. 2012;38(2):272-278.

22. Ahn DH, Illum HB, Wang DH, Sharma A, Dowell JE. Upper extremity venous thrombosis in patients with cancer with peripherally inserted central venous catheters: a retrospective analysis of risk factors. J Oncol Pract. 2013;9(1):e8-e12.

23. Debourdeau P, Kassab Chahmi D, Le Gal G, et al. 2008 Standards, Options and Recommendations (SOR) guidelines for the prevention 
and treatment of thrombosis associated with central venous catheters in patients with cancer: report from the working group. Ann Oncol. 2009;20(9):1459-1471.

24. Greene MT, Flanders SA, Woller SC, Bernstein SJ, Chopra V. The association between picc use and venous thromboembolism in upper and lower extremities. Am J Med. 2015;128(9):986-993.e1.

25. Kaplan D, Casper TC, Elliott CG, et al. VTE Incidence and risk factors in patients with severe sepsis and septic shock. Chest. 2015; 148(5):1224-1230.

26. Infusion Nurses Society. Infusion nursing standards of practice. I Infus Nurs. 2011;34(1S).

27. O'Grady NP, Alexander M, Burns LA, Dellinger EP, Garland J, Heard SO, et al. Healthcare Infection Control Practices Advisory Committee (HICPAC) (Appendix 1). Summary of recommendations: Guidelines for the Prevention of Intravascular Catheter-related Infections. Clin Infect Dis. 2011;52:1087-1099.

28. Mollee P, Jones M, Stackelroth J, et al. Catheter-associated bloodstream infection incidence and risk factors in adults with cancer: a prospective cohort study. J Hosp Infect. 2011;78(1):26-30.

29. Safdar N, Maki DG. Risk of catheter-related bloodstream infection with peripherally inserted central venous catheters used in hospitalized patients. Chest. 2005;128(2):489-495.

30. O'Grady NP, Alexander M, Burns LA, et al. Guidelines for the prevention of intravascular catheter-related infections. Clin Infect Dis. 2011;52(9):e162-e193.

31. Tejedor SC, Tong D, Stein J, et al. Temporary central venous catheter utilization patterns in a large tertiary care center: tracking the "idle central venous catheter". Infect Control Hosp Epidemiol. 2012;33(1): 50-57.

32. Gibson C, Connolly BL, Moineddin R, Mahant S, Filipescu D, Amaral JG. Peripherally inserted central catheters: use at a tertiary care pediatric center. J Vasc Interv Radiol. 2013;24(9):1323-1331.

33. Chopra V, Govindan S, Kuhn L, et al. Do clinicians know which of their patients have central venous catheters?: a multicenter observational study. Ann Intern Med. 2014;161(8):562-567.

34. Choosing Wisely. American Society of Nephrology. Don't place peripherally inserted central catheters (PICC) in stage III-V CKD patients without consulting nephrology. Available at: http://www. choosingwisely.org/clinician-lists/american-society-nephrology- peripherally-inserted-central-catheters-in-stage-iii-iv-ckd-patients. Accessed November 3, 2015.

35. Society of General Internal Medicine. Don't place, or leave in place, peripherally inserted central catheters for patient or provider convenience. Available at: http://www.choosingwisely.org/clinician-lists/society-general-internal-medicine-peripherally-inserted-central-cathetersfor-patient-provider-convenience. Accessed November 3, 2015.

36. Fitch K, Bernstein SJ, Aguilar MD, et al. The RAND/UCLA appropriateness method user's manual. Santa Monica, CA: RAND; 2001. Available at: http://www.rand.org/pubs/monograph_reports/MR1269. html.

37. National Kidney Foundation/Kidney Disease Outcomes Quality Initiative. KDOQI 2012 clinical practice guidelines for chronic kidney disease. Kidney Inter. 2013; (suppl 3):1-150. Accessed November 3, 2015.

38. Rupp SM, Apfelbaum JL, Blitt C, et al. Practice guidelines for central venous access: a report by the American Society of Anesthesiologists Task Force on Central Venous Access. Anesthesiology. 2012;116(3): 539-573.

39. Robinson MK, Mogensen KM, Grudinskas GF, Kohler S, Jacobs DO. Improved care and reduced costs for patients requiring peripherally inserted central catheters: the role of bedside ultrasound and a dedicated team. JPEN J Parenter Enteral Nutr. 2005;29(5):374-379.

40. Trerotola SO, Thompson S, Chittams J, Vierregger KS. Analysis of tip malposition and correction in peripherally inserted central catheters placed at bedside by a dedicated nursing team. J Vasc Interv Radiol. 2007;18(4):513-518.

41. Food and Drug Administration Task Force. Precautions necessary with central venous catheters. FDA Drug Bull. 1989:15-16.

42. O’Brien J, Paquet F, Lindsay R, Valenti D. Insertion of PICCs with minimum number of lumens reduces complications and costs. $J \mathrm{Am}$ Coll Radiol. 2013;10(11):864-868.

43. Dal Molin A, Allara E, Montani D, et al. Flushing the central venous catheter: is heparin necessary? J Vasc Access. 2014;15(4):241-248.

44. Lopez-Briz E, Ruiz Garcia V, Cabello JB, Bort-Marti S, Carbonell Sanchis R, Burls A. Heparin versus $0.9 \%$ sodium chloride intermittent flushing for prevention of occlusion in central venous catheters in adults. Cochrane Database Syst Rev. 2014;10:CD008462. 